\title{
Evaluation of cumulative damage in glass fibre reinforced plastic by ultrasonic method
}

\author{
V K SRIVASTAVA and R PRAKASH* \\ Department of Mechanical Engineering, *Department of Bio-Medical Engineering, \\ Institute of Technology, Banaras Hindu University, Varanasi 221005 , India \\ MS received 20 November 1986; revised 16 December 1986

\begin{abstract}
This paper presents the cumulative damage in glass fibre reinforced plastic composite during loading and unloading at regular intervals using the continuous monitoring of ultrasonic test instrument. The result shows that the ultrasonic attenuation increases with an increase in the tensile load, which provides the change in internal damage of composites.
\end{abstract}

Keywords. Cumulative damage; E-glass fibre; epoxy resin; ultrasonic technique; hounsfield tensemeter; tensile load.

\section{Introduction}

Ultrasonic non-destructive testing is based on the interaction of sound waves passing through a material with the structure of the material. If a material is homogeneous and of a sufficiently good quality, a sound wave will pass through it with a relatively little loss of energy. If the material is not homogeneous (i.e. if there are cracks or voids within it), the sound wave gets scattered and/or reflected at these features giving rise to (i) a greater attenuation of the transmitted beam and (ii) the presence of reflected and/or scattered waves. If a wave is to be appreciably scattered by any scattering centre the wave must have a wavelength of the order of the size of scattering centre or less (Prakash 1980). Stone and Clarke (1975) evaluated the void content by ultrasonic technique in carbon fibre reinforced plastics (CFRP). Their results show that ultrasonic attenuation is not very sensitive to fibre volume fraction. Interlaminar cracks are also occasionally present in fibre reinforced plastic composites, also ultrasonic attenuation in the same way, as voids do. Birchon (1972) tried to differentiate between voids and interlaminar cracks by observing the ultrasonic responses from these two features. Knollman et al (1979) determined the cumu-. lative internal damage in filled polymers using an ultrasonic technique. Hayford et al (1977) presented a correlation between ultrasonic attenuation and shear strength of graphite-polyimide composite samples. Their results showed a good correlation between the initial ultrasonic attenuation values of the samples and the shear strength of these samples. The present paper describes the ultrasonic detection of cumulative damage in glass fibre reinforced plastic composite.

\section{Experimental procedure}

Glass fibre reinforced plastic (GRP) composites were made by hand lay-up technique. The composites $\left(0^{\circ} / 90^{\circ}\right.$ and $\left.\pm 45^{\circ}\right)$ were moulded by using the E-glass 
fibre woven mats and epoxy resin (CY-205 resin and HY-951 hardner). Epoxy resin sheets were also moulded for the present study. These GRP and epoxy resin sheets were cut to obtain flat specimens for experimentation. The test set-up used for evaluating cumulative damage in GRP composites is shown in figure 1. Tensile loading was applied using commercially available Hounsfield tensometer. A water bath made of "Perspex" fabricated sheet was used so as to fit in the tensometer and keep the gauge length portion of specimen submerged in water. The transmitter and receiver probes $(10 \mathrm{MHz}$ frequency) were placed on the top and bottom of the specimen and the water bath was filled with water to provide a medium for the transmission of ultrasonic energy from transmitter probe to receiver probe through the specimen. The ultrasonic attenuation values were continuously monitored by recording the peak heights of the ultrasonic signal corresponding to various loads for epoxy specimens, $0^{\circ} / 90^{\circ}$ cross-ply GRP specimens and $\pm 45^{\circ}$ GRP specimens. The specimens were loaded maximum upto $40 \%$ of the ultimate tensile strength value for GRP composites. Readings were taken at regular intervals during loading and unloading of the specimen.

\section{Results and discussion}

Curves were plotted to show the variation of ultrasonic attenuation with the increasing/decreasing tensile load for epoxy specimens (figure 2), $0^{\circ} / 90^{\circ}$ cross-ply GRP specimens (figure 3) and $\pm 45^{\circ}$ GRP specimens (figure 4). These curves show that ultrasonic attenuation increases with increasing load from the very beginning and on unloading, the attenuation values start decreasing but they do not retrace their path (i.e. the value of ultrasonic attenuation (during unloading) does not remain what it was for a particular load, during increasing load cycle). On complete unloading, the new ultrasonic attenuation value is higher than the one previously obtained for the same material, until and unless some microdamage takes place within the material. Since ultrasonic attenuation is a material parameter, it should not change. On further loading, the ultrasonic attenuation values once again starts rising and again, on unloading the values start falling as was the trend during the first loading/unloading cycle. At the end of this second cycle the ultrasonic attenuation of the material once again was higher than the previous two ultrasonic attenuation values (points $A$ and $B$ in figure 2). This clearly indicates that further microdamage has crept-in. In other words, the measured ultrasonic attenuation may provide the specimens under tests. For GRP specimens also, a behaviour similar to that for epoxy specimens was observed. Figure 3 shows the tensile loading vs ultrasonic attenuation curve for $0^{\circ} / 90^{\circ}$ cross-ply GRP specimens and the same trend was also recorded for $\pm 45^{\circ}$ GRP specimens (figure 4). Our results show that ultrasonic attenuation increases with increasing tensile load, since ultrasonic attenuation is related to the amount of defects present in the material under test (Stone and Clarke 1975). It is almost certain that the loading of the specimen causes microdamages within the specimen. This damage may be in the form of a resin crazing, resin cracking or microcracks in weak fibre etc. Whatever may be the cause for higher ultrasonic attenuation, it points to the fact that the damage within the material has increased. 


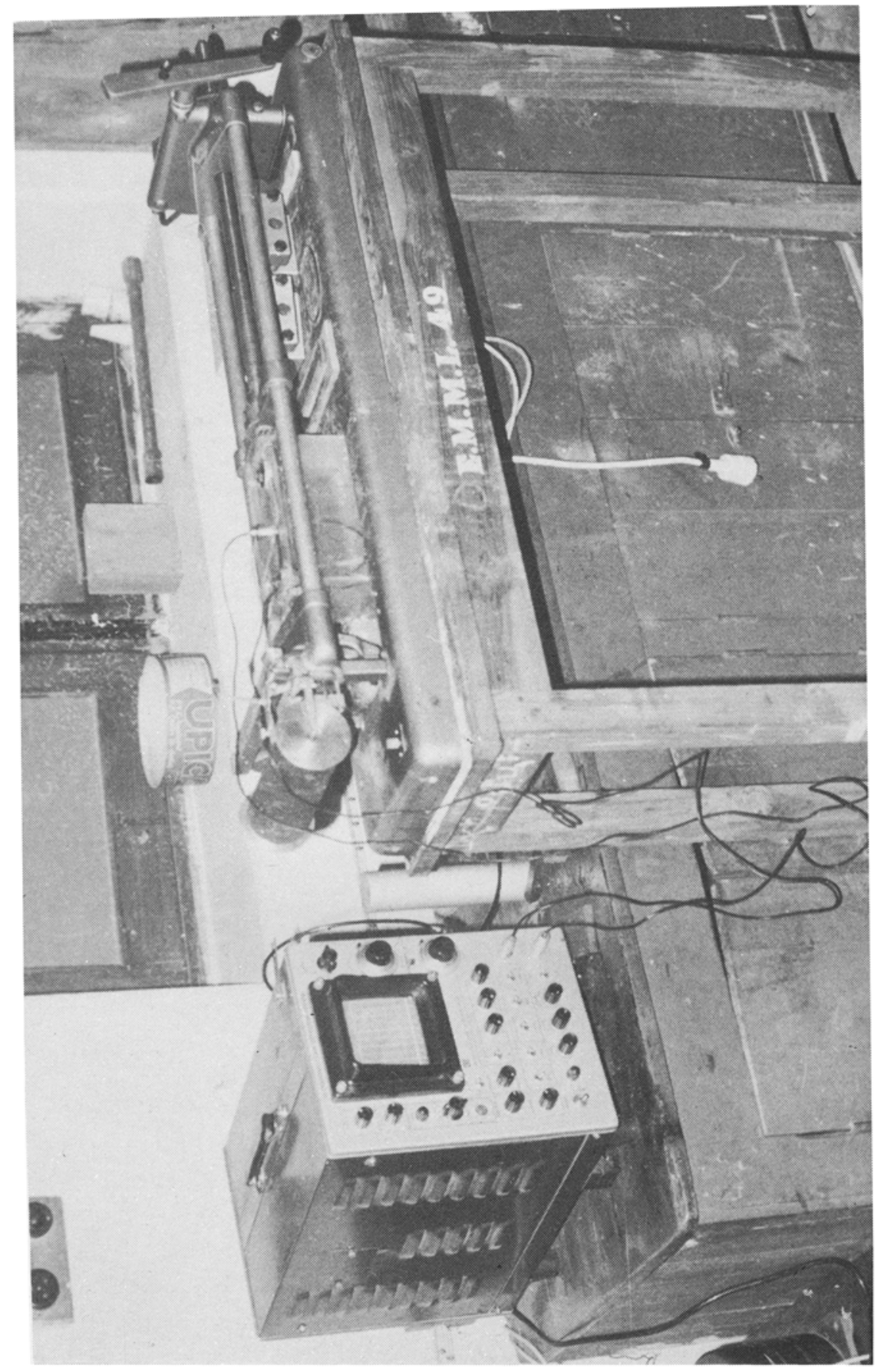

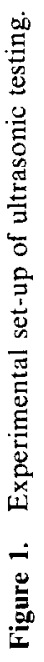




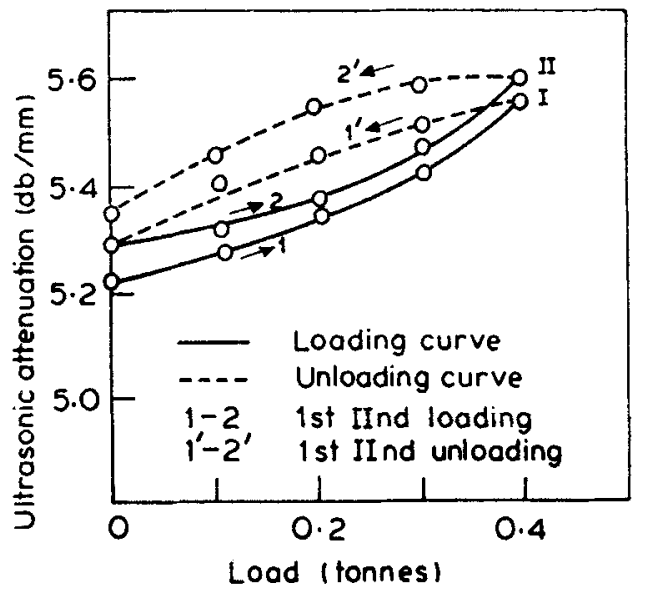

Figure 2. Ultrasonic attenuation vs tensile load of epoxy.

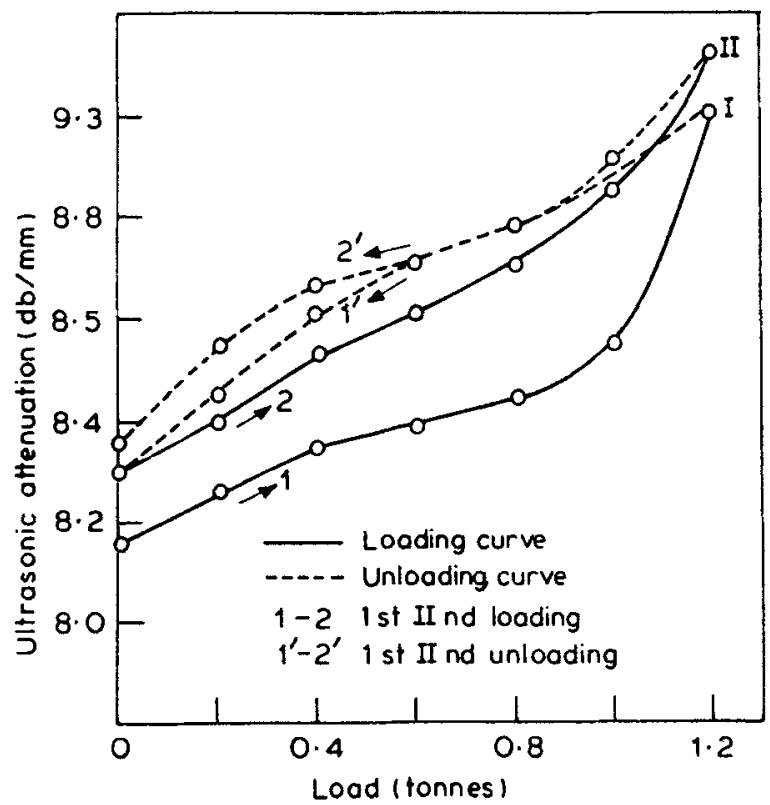

Figure 3. Ultrasonic attenuation vs tensile load of $0^{\circ} / 90^{\circ}$ cross-ply GRP composites.

\section{Conclusion}

The study on cumulative damage in GRP composites by ultrasonic technique proves that the change in ultrasonic attenuation is due to microdamages within the material. However, the change in ultrasonic attenuation serves as a measure of cumulative damage in GRP composites i.e. $\Delta \alpha=\left(\alpha_{u}-\alpha_{i}\right) \mathrm{db} / \mathrm{mm}$ is proportional to the cumulative damage in GRP composites where $\alpha_{u}$ is the ultrasonic attenuation after loading/unloading cycle and $\alpha_{i}$ is the initial ultrasonic attenuation. 


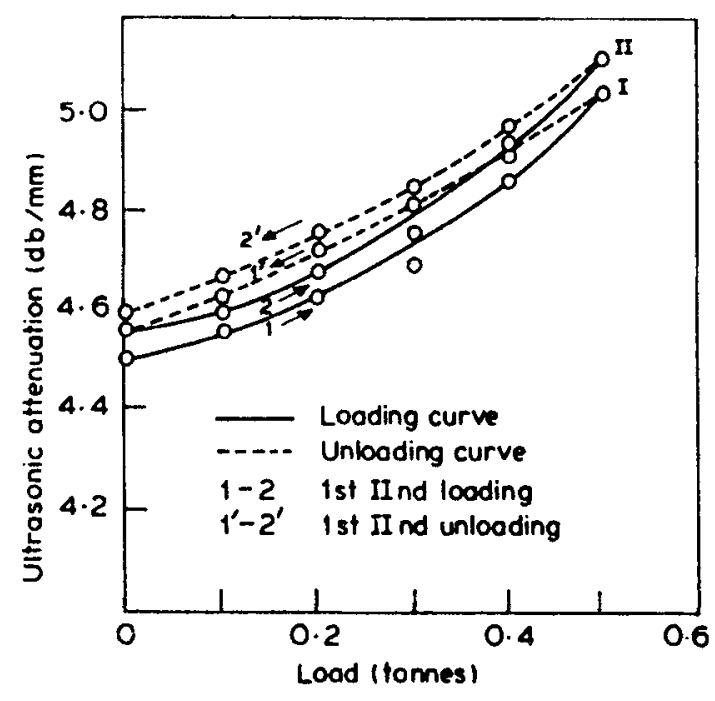

Figure 4. Ultrasonic attenuation vs tensile load of $\pm 45^{\circ}$ cross-ply GRP composites.

\section{References}

Birchon D 1972 J. Chart. Mech. Eng. 1754

Hayford D T, Henneke II E G and Stinchcomb 1977 J. Composite Mater. 11429

Knollman G C, Martinson R H and Bellin J L 1979 J. Appl. Phys. 50111

Prakash R 1980 J. Compos. 9217

Stone D E W and Clarke B 1975 J. Non Destructive Testing 7137 